\title{
Frequency and Antimicrobial Sensitivity Pattern of Extended Spectrum $\beta$-Lactamases Producing Escherichia coli and Klebsiella pneumoniae Isolated from urine at a Tertiary Care Hospital
}

\author{
Islam MB ${ }^{1}$, Jhora $\mathrm{ST}^{2}$, Yusuf MA ${ }^{3}$, Sattar $\mathrm{AFMA}^{4}$, Shahidullah $\mathrm{SK}^{5}$, Rahman NMW ${ }^{6}$, Sharif $\mathrm{AR}^{7}, \mathrm{Ahsan} \mathrm{AI}^{8}$, \\ Wadud $\mathrm{ABMA}^{9}$, Chowdhury $\mathrm{MS}^{10}$
}

\begin{abstract}
Background: Infections due to extended spectrum $\beta$-lactamases (ESBL) producing Escherichia coli and Klebsiella pneumoniae have become an important clinical problem. These organisms are important regarding the infection control by the physicians. Objective: The present study was undertaken to determine the prevalence of ESBLs along with their antimicrobial sensitivity pattern in Escherichia coli and Klebsiella pneumoniae. Methodology: This cross sectional study was conducted in the Department of Microbiology at Sir Salimullah Medical College, Dhaka. Urine samples were collected from patients who were clinically suspected to have UTI. After incubation, plates were checked for presence of suspected pathogens. Organisms were identified to species level by conventional methods. All isolated E. coli and $K$. pneumoniae were included in the study. The susceptibility to antibiotics was determined by Kirby Bauer method on Muller Hinton agar. Isolates were screened for ESBL production by using disk diffusion of cefotaxime, ceftazidime, ceftriaxone and cefpodoxime placed on inoculated plates containing Muller Hinton agar according to the CLSI recommendations. Phenotypic confirmatory test for ESBL producers was done by combined disc diffusion for all the isolates that were screened positive for the ESBL production following CLSI guidelines. Combined disk diffusion method was also done in this study. Result: A total of 220 non repeated urine samples were cultured of which 132(60\%) cases had shown the bacterial growth. Among the 132 samples Escherichia coli had found in 103(78.0\%) cases and Klebsiella spp. was found in $14(10.6 \%)$ cases. Out of 103 E coli 23(22.3\%) cases was found as ESBL strain. On the other hand within 14 Klebsiella species, the ESBL strain was found in 5(35.7\%) cases. Both E coli and Klebsiella species were $100 \%$ sensitive to imipenem. However, cephamycin was sensitive in $93.7 \%$ and $100 \%$ in E coli and Klebsiella species respectively. Conclusion: Results indicate that routine ESBL detection should be made imperative and empirical use of third generation cephalosporins must be discouraged.
\end{abstract}

Key words: Extended spectrum $\beta$-lactamases, Escherichia coli, Klebsiella pneumoniae, 3rd generation cephalosporin

\section{Introduction}

The most common cause of bacterial resistance to $\beta$-lactam antibiotics is the production of $\beta$-lactamases ${ }^{1}$. The latest in the arsenal of these enzymes has been the evolution of extended spectrum $\beta$-lactamases (ESBLs). ESBLs are defined as $\beta$-lactamases capable of hydrolyzing oxyiminocephalosporins and are inhibited by $\beta$-lactamase inhibitors $^{2}$. An extensive use of $\beta$-lactam antibiotics in hospitals and community has created major resistance problem leading to increased morbidity, mortality and health-care costs ${ }^{3}$.

The incidence of ESBL producing strains among clinical isolates has been steadily increasing over the past years resulting in limitation of therapeutic options ${ }^{4}$. Microorganisms responsible for urinary tract infection (UTI) such as E. coli and Klebsiella spp. have the ability to produce ESBLs in large quantities. These enzymes are plasmid borne and confer multiple drug resistance, making

1. Dr. Md. Badrul Islam, Associate Professor, Department of Microbiology, Dhaka National Medical College, Dhaka

2. Prof. Sanya Tahmina Jhora, Professor \& Head, Department of Microbiology, Sir Salimullah Medical College, Dhaka

3. Dr. Md. Abdullah Yusuf, Lecturer, Department of Microbiology, Shaheed Suhrawardy Medical College, Dhaka

4. Dr. AFM Arshedi Sattar, Clinical Pathologist, Dhaka Medical College Hospital, Dhaka

5. Dr. Sk Shahidullah, Assistant Professor, Department of Microbiology, NICVD, Dhaka

6. Dr. NM Wahidur Rahman, Assistant Professor, Department of Microbiology, Eastern Medical College, Comilla

7. Dr. Ahmed Raihan Sharif, Medical Officer, Department of Virology, IEDCR, Dhaka

8. Dr. Ali Imam Ahsan, Junior Consultant, SAHIK, Dhaka

9. Dr. ABM Abdul Wadud, Consultant, Microbiology \& Immunology, LABAID Group, Dhaka

10. Dr. Md. Shahjahan Chowdhury, Associate Professor, Department of Pathology, Dhaka National Medical College, Dhaka

\section{Correspondence}

Dr. Md. Badrul Islam, Associate Professor, Department of Microbiology, Dhaka National Medical College, Dhaka; Email : bodrulislam63@gmail.com; Mobile: +8801670738692 
urinary tract infection difficult to treat ${ }^{5}$.

Extended spectrum $\beta$-lactamases are a large, rapidly evolving group of plasmid mediated enzymes capable of hydrolyzing and inactivating penicillins, cephalosporins and monobactams and are inhibited by $\beta$-lactamase inhibitors such as clavulanate, sulbactam and tazobactam $^{6,7,8}$. Since their description in the mid-1980s, ESBLs spread rapidly to Europe, US and Asia and are now found all over the world ${ }^{9}$. They are also involved in nosocomial outbreaks conferring multiple drug resistant and resulting in limitation of therapeutic options ${ }^{10,11}$. Specific risk factors that have led to spread of ESBL include prolonged hospitalization, severity of illness, intubations and mechanical ventilation, urinary or arterial catheterization and extensive use of broad spectrum antibiotics $^{12,13}$. Plasmid genes are easily transferred among enterobacteria, contributing to ESBL dissemination ${ }^{14}$. Plasmids that carry $\beta$-lactamase genes frequently harbour resistance genes to other antimicrobials ${ }^{15}$. Therefore, the detection of ESBL-producing isolates is critical to assure appropriate therapy and to prevent their dissemination. This study was initiated to identify the incidence of ESBL producers in urinary isolates of E. coli and $K$. pneumoniae and also to see the pattern of susceptibility of the isolates to other clinically relevant antimicrobials. The aim of the present study was to identify the freaqency of ESBL producing E. coli \& Klebsiella spp. with their artibiogram.

\section{Methodology}

This study was conducted from January to December 2006 in the Department of Microbiology at Sir Salimullah Medical College, Dhaka. A total of 220 urine samples were collected from patients who were clinically suspected to have UTI. Both the outpatients and inpatients were included in the study. All urine samples were inoculated in Blood agar and MacConkey agar media. All the plates were incubated at $37^{\circ} \mathrm{C}$ aerobically for 24 hours. After incubation, plates were checked for presence of suspected pathogens. Organisms were identified to species level by conventional methods $^{16}$. All isolated E. coli and K. pneumoniae were included in the study. Clinico-demographic data of the study patients was noted.

Antimicrobial susceptibility testing: The susceptibility to antibiotics was determined by Kirby Bauer method on Muller Hinton agar media according to $\mathrm{CLSI}^{17}$ protocols. The drugs tested were Ampicillin $(10 \mu \mathrm{g})$, Amoxycillinclavulanic acid $(20 / 10 \mu \mathrm{g})$, Piperacillin $(100 \mu \mathrm{g})$, Piperacillin -tazobactam $(100 / 10 \mu \mathrm{g})$, Cephotaxime $(30 \mu \mathrm{g})$, Ceftriaxone $(30 \mu \mathrm{g})$, Ceftazidime $(30 \mu \mathrm{g})$, Cefpodoxime $(10 \mu \mathrm{g})$, Gentamicin $(10 \mu \mathrm{g})$, Amikacin $(30 \mu \mathrm{g})$, Ciprofloxacin $(5 \mu \mathrm{g})$, Tetracycline $(30 \mu \mathrm{g})$, Chloramphenicol $(30 \mu \mathrm{g})$, Trimethoprim-sulfamethoxazole $(1.25 / 23.7 \mu \mathrm{g})$ and Imipenem $(10 \mu \mathrm{g})$. E. coli ATCC 25922 was used as control strains.
Screening for ESBLs: Isolates were screened for ESBL production by using disk diffusion of cefotaxime, ceftazidime, ceftriaxone and cefpodoxime placed on inoculated plates containing Muller Hinton agar media according to the $\mathrm{CLSI}^{17}$ recommendations. Isolates showing inhibition zone size of $22 \mathrm{~mm}$ with ceftazidime $(30 \mu \mathrm{g})$, $25 \mathrm{~mm}$ with ceftriaxone $(30 \mu \mathrm{g}), 27 \mathrm{~mm}$ with cefotaxime $(30 \mu \mathrm{g})$ and $17 \mathrm{~mm}$ for cefpodoxime were suspected for ESBL production. E. coli ATCC 25922 was used as a negative control.

Confirmatory test for ESBLs: Phenotypic confirmatory test for ESBL producers was done by combined disc diffusion for all the isolates that were screened positive for the ESBL production following CLSI ${ }^{17}$ guidelines.

Combined disk diffusion method: In this test a disk of ceftazidime $(30 \mu \mathrm{g})$ alone and a disk of ceftazidime in combination with clavulanic acid $(30 / 10 \mu \mathrm{g})$ were used. Both the disks were placed $25 \mathrm{~mm}$ apart, centre to centre, on a lawn culture of the test isolate on Muller Hinton agar plate and was incubated overnight at $37^{\circ} \mathrm{C}$. Difference in zone diameter with and without clavulanic acid was measured. The positive result was defined as $5 \mathrm{~mm}$ increase in inhibition zone diameter around combination disks with clavulanic acid versus its standard zone when tested alone ${ }^{17}$.

\section{Results}

Out of the 220 consecutive, non-repeat urine samples processed, 132(60.0\%) samples yielded various bacterial isolates. Among them, E. coli were the highest number of isolated from the specimen which was $103(78.0 \%)$ and the next to this is the $K$. Pneumoniae which was isolated in $14(10.6 \%)$ cases. ESBL production was observed in $22.3 \%$ of $E$. coli (23/103) and $35.7 \%$ of $K$. pneumoniae (5/14).

Table 1 : Age distribution among the study population $(n=28)$

\begin{tabular}{lcc}
\hline Age group & Frequency & Percentage \\
\hline$<20$ & 3 & 10.7 \\
$20-40$ & 19 & 67.8 \\
$40-60$ & 5 & 17.9 \\
$>60$ & 1 & 3.6 \\
Total & $\mathbf{2 8}$ & $\mathbf{1 0 0}$ \\
\hline
\end{tabular}

*Mean age $\pm \mathrm{SD}=35 \pm 24.7$

All of them showed inhibition zone size of $22 \mathrm{~mm}$ with ceftazidime during screening test. Confirmatory test for ESBL production were performed by $\mathrm{CLSI}^{17}$ confirmatory test on these 28 isolates of E. coli and Klebsiella pneumoniae. These ESBL positive isolates were obtained from 12 male and 16 female patients with a male female ratio of 1:1.3. They were distributed in the age group of 1 month to 77 years and the mean age of the study population was $35 \pm 24.7$ years. The antimicrobial susceptibility results of 
ESBL producers were also done and showed that susceptibility of ESBL producers to imipenem, nitrofurantoin and amikacin were found to be $100 \%, 89 \%$ and $86 \%$ respectively.

Table 2 : Distribution of sex among the study population $(n=28)$

\begin{tabular}{lcc}
\hline Sex group & Frequency & Percentage \\
\hline Male & 12 & 42.9 \\
Female & 16 & 57.1 \\
Total & $\mathbf{2 8}$ & $\mathbf{1 0 0}$ \\
\hline
\end{tabular}

*Male $:$ Female $=1: 1.3$

The antimicrobial resistance was significantly higher in ESBL producers than in non-ESBL producers. ESBL producers were almost always resistant to ampicillin and piperacillin. Both E coli and Klebsiella species were 100\% sensitive to imipenem. However, cephamycin was sensitive in $93.7 \%$ and $100 \%$ in E coli and Klebsiella species respectively.

Table 3 : Distribution ESBL producing $E$ coli and Klebsiella species $(n=117)$

\begin{tabular}{lccc}
\hline $\begin{array}{l}\text { Bacteria } \\
\text { Isolated }\end{array}$ & $\begin{array}{c}\text { ESBL } \\
\text { positive }\end{array}$ & $\begin{array}{c}\text { ESBL } \\
\text { negative }\end{array}$ & Total \\
\hline E coli & $23(82.1 \%)$ & $80(89.9 \%)$ & $103(88.0 \%)$ \\
Klebsiella & $5(17.9 \%)$ & $9(10.1 \%)$ & $14(12.0 \%)$ \\
species & & & \\
Total & $\mathbf{2 8 ( 1 0 0 \% )}$ & $\mathbf{8 9}(\mathbf{1 0 0 \% )}$ & $\mathbf{1 1 7 ( 1 0 0 \% )}$ \\
\hline
\end{tabular}

* Pearson Chi-Square test was corrected by Fisher's Exact Test $*$ p value $=0.318$

Cephalosporin resistance was also higher in ESBL producing $E$. coli and $K$. pneumoniae isolates when compared to ESBL non producers. Combination of $\beta$ lactam/ $\beta$-lactamase inhibitors showed greater activity in both ESBL producers and non producers. Among aminoglycosides, amikaicn showed greater activity against all the isolates irrespective of their ESBL status.

\section{Discussion}

Antibiotic resistance monitoring has a central role among all strategies to manage the problem of antibiotic policy. Since their first description in the mid 1970s, ESBLs have been isolated worldwide and form a major contributor of drug resistance in many of Enterobacteriaceae. ESBLs are now a problem in hospitalized patients throughout the world $^{11}$. The prevalence of ESBLs among clinical isolates vary greatly world wide and in geographic areas and are rapidly changing overtime ${ }^{18}$. Of the 132 strains included in this study $23.5 \%$ showed ESBL production, with the highest incidence in E. coli $(82.1 \%)$ followed by $K$. pneumoniae $(17.9 \%)$. The ESBL production is alarming. The incidence of ESBL in major hospitals of India has been reported to be as high as $58 \%{ }^{19}$. The range of ESBL isolation rate has been varied from 6 to $39 \%$ in different studies $^{20,21,22,23}$. However, very similar percentage was reported from Chennai ${ }^{24}(20 \%)$ and Hyderabad ${ }^{25}$ (19.8\%). One reason for such variability may be the very low number of samples studied.

Interestingly among all ESBL isolates, it is predominantly present among E. coli (82.1\%) compared to K. pneumoniae (17.9\%). Similar finding showing a high prevalence of ESBLs among $E$. coli ${ }^{25,26}$ was reported. The high incidence of ESBLs among E. coli may be peculiar to the Indian subcontinent. Cefpodoxime showed the highest sensitivity in detecting ESBL producing E. coli and K. pneumoniae as reported earlier. Organisms that express an ESBL are frequently resistant to other antimicrobial agents, as many of these additional resistant genes are encoded on the ESBL associated plasmid ${ }^{27}$. In this study high level of resistance was observed against tetracycline. However, good activity was showed by $\beta$-lactam/ $\beta$-lactamase inhibitor combination. Among the non $\beta$-lactam antibiotics, amikacin showed higher sensitivity against these ESBL producers. Similar results were reported for the patients with serious infections with ESBL producers ${ }^{28}$. In the present study, ESBL producing isolates were isolated from inpatients units as well as from clinical samples from patients attending outpatient. As indicated in many previous studies all ESBL producers were found to be susceptible to imipenem and amikacin. However, amikacin and carbapenems are usually used only as the reserve drugs. A similar study conducted by et $\mathrm{al}^{20}$ and Abigail et $\mathrm{al}^{29}$ showed $100 \%$ susceptibility to amikacin and imipenem. The marked increase in $\beta$-lactamase production, including the high level constitutive ESBL producers have

left us with few alternatives in combating serious infection.

\section{Conclusion}

In conclusion, this study emphasizes the need for continued surveillance of ESBL producing bacteria as high prevalence of antibiotic resistance in ESBL positive $E$. coli and $K$. pneumoniae was observed. Phenotypic confirmatory test using combination disk is simple and cost effective for the detection of ESBL producers as it has $100 \%$ concordance with MIC reduction test. The control measure include judicious use of antibiotics, strict hygiene protocols and implementation of appropriate infection control measures in the hospital, especially while treating high risk patients.

\section{References}

1. Sasirekha B, Manasa R, Ramya P, Sneha R. Frequency and Antimicrobial Sensitivity Pattern of Extended Spectrum $\beta$-Lactamases Producing E. coli and Klebsiella pneumoniae Isolated In A Tertiary Care Hospital. Al Ameen J Med Sci 2010;3(4):265-271

2. Bush K, Jacoby GA, Medeiros AA. A functional classification scheme for $\beta$ - lactamases and its correlation with molecular structure. Antimicrob Agents Chemother 1995;39:1211-1233

3. Maiti SN, Phillips OA, Micetich RG, Livermore DM. Beta-lactamase inhibitors: Agents to overcome bacterial resistance. Curr Med Chem 1998; 5:441-456

4. Podschun R, Ullmann U. Klebsiella species as Nosocomial Pathogens: 
epidemiology taxonomy, typing methods and pathogenicity factors. Clin Microbiol Rev 1998; 11:589-603

5. Bal S. $\beta$-lactamase mediated resistance in hospital-acquired Urinary Tract Infections. Hospital Today 2000;5:96-101

6. Ndugulile F, Jureen R, Harthug S, Urassa W, Langeland N. Extended spectrum $\beta$-lactamases among Gram-negative bacteria of nosocomial origin from an Intensive Care Unit of a tertiary health facility in Tanzania. BMC Infect. Dis $2005 ; 5: 86-97$

7. Spanu T, Sanguinetti M, Tumbarello M, D'Inzeo T, Fioir B, Posteraro B et al. Evaluation of the new VITEK 2 extended-spectrum beta-lactamase (ESBL) test for rapid detection of ESBL production in Enterobacteriaceae isolates. J Clin Microbiol 2006; 44(9):3257-3262

8. Hosoglu S, Gundes S, Kolayli F, Karadenizil A, Demirdag K, Gunaydin $\mathrm{M}$ et al. Extended-spectrum beta-lactamases in Ceftazidime-resistant Escheriachia coli and Klebsiella pneumoniae isolates in Turkish hospitals. Indian J Med Microbiol 2007;25(4):346-350

9. Revathi G, Singh S, Simrita S. Detection of expanded spectrum cephalosporin resistance due to inducible lactamases in hospital isolates. Indian J Med Microbiol 1997; 15(3):113-115

10. Paterson DL, Ko WC, Gottberg V, Mohapatra S, Casellas JM, Goossens $\mathrm{H}$ et al. Antibiotic therapy for Klebsiella pneumoniae bacteriamia: implications of production of extended-spectrum $\beta$-lactamases. CID 2004; 39 (1): 31-37

11. Canton R, Novais A, Valverde A, Machado E, Peixe L, Baquero F et al. Prevalence and spread of extended-spectrum $\beta$-lactamase producing Enterobacteriaceae in Europe. CMI. 2008; 14(1):144-153

12. Lin MF, Huang ML, Lai SH. Risk factors in the acquisition of extendedspectrum beta-lactamase Klebsiella pneumoniae: a case control study in a district teaching hospital in Taiwan. J Hosp Infect 2003; 53:39-45

13. Tumbarello M, Sanguinetti M, Montuori E, Tercarichi EM, Posteraro B, Fiori B et al. Predictors of mortality in patients with blood stream infections caused by extended-spectrum $\beta$-lactamase producing Enterobacteriaceae: Importance of inadequate initial antimicrobial treatment. Antimicrob Agents Chemother 2007;51(6):1987-1994

14. Gruteka P, Goessens W, Gils J, Peerbooms P, Toom NL, Verheuvel MS et al. Patterns of resistance associated with integrons,the extended spectrum $\beta$-lactamase SHV-5 gene, and a multidrug efflux pump of Klebsiella pneumoniae causing a nosocomial outbreak. J Clin Microbiol 2003;41(3):1161-1166

15. Mulvey MR, Soule G, Boyd D, Demczuk W, Ahmed R. Characterization of the first extended-spectrum beta-lactamase producing Salmonella isolate identified in Canada. J Clin Microbiol 2003; 41(1):460-462

16. Koneman EW, Allen SD, Janda WM, Shcreckenberger PC, Win WC. The Enterobacteriaceae. In: color atlas and textbook of diagnostic microbiology, 5th ed. JB Lippincott Co: Philadelphia, 2006; 211-302

17. Clinical Laboratory Standards Institute (CLSI). Performance standards for antimicrobial susceptibility testing. 16th informational supplement .2006; M100-S15

18. Livermore DM. $\beta$-Lactamases in laboratory and clinical resistance. Clin Microbiol Rev 1995; 8:557-584

19. Mathur P, Kapil A, Das B, Dhawan B. Prevalence of extended spectrum $\beta$-lactamase producing Gram negative bacteria in a tertiary care hospital. Indian J Med Res 2002; 115:153-157

20. Hansotia JB, Agarwal V, Pathak AA, Saoji AM. Extended spectrum $\beta$ lactamase mediate resistance to third generation cephalosporins in Klebsiella pneumoniae in Nagpur, central India. Indian J Med Res 1997; 105:160-165

21. Manchanda V, Singh NP, Goyal R, Kumar A, Thukral SS. Phenotypic characteristics of clinical isolates of Klebsiella pneumoniae and evaluation of available techniques for detection of extended spectrum beta lactamases. Indian J Med Res 2005; 122:330-337

22. Tankhiwale SS, Jalgaonkar SV, Ahamad S, Hassani U. Evaluation of extended spectrum beta lactamase in urinary isolates. Indian J Med Res 2004; 120:553-556
23. Jain A, Roy I, Gupta MK, Kumar M, Agarwal SK. Prevalence of extended spectrum beta lactamase producing Gram negative bacteria in septicaemia neonates in a teritary care hospital. J Med Microbio 2003; 52:421-425

24. Kumar MS, Lakshmi V, Rajagopaln R. Occurrence of Extended spectrum bet lactamases among Enterobacteriaceae species isolated at a teritary care institute. Indian J Med Microbiol 2006; 24 (3):208-211

25. Menon T, Bindu D, Kumar CPG, Nalini S, Thirunarayan MA. Comparison of double disc and three dimensional methods to screen for ESBL producers in a tertiary care hospital. Indian J Med Microbiol 2006; 24 (2):117-120

26. Ananthakrishnan AN, Kanungo R, Kumar A, Badrinath S. Detection of extended spectrum $\beta$-lactamase producers among surgical wound infections and burn patients in JIPMER. Indian J. Med Microbiol 2004; 18:160-165

27. Babypadmini S, Appalaraju B. Extended spectrum-lactamases in urinary isolates of Escherichia coli and Klebsiella pneumoniae-Prevalence and susceptibility pattern in a tertiary care hospital. Indian J Medical Microbiology 2004;22(3):172-174

28. Zoltan P, Zsofia K, Elisabeth N. Characterization of extended-spectrum $\beta$ - lactamases and determination of the antibiotic susceptibility of Klebsiella pneumoniae isolates in Hungary. J. Antimicrob Chemother 1998; 42: 401-403 29. Abigail S, Mathai E, Jesudasan MV, Jhon TJ. Ceftazidime resistance among Klebsiella pneumoniae in South India. Indian J Med Res 1995; 102:53-88 